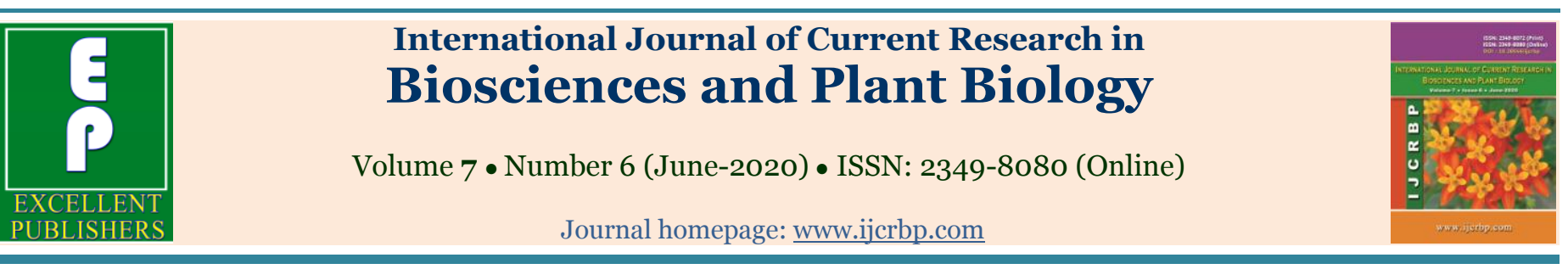

\title{
Sacred groves of Point Calimere Wildlife and Bird Sanctuary - Ideal centres for biodiversity conservation
}

\author{
M. Padma Sorna Subramanian'1, A. Saravana Ganthi ${ }^{2 *}$ and K. Subramonian ${ }^{3}$ \\ ${ }^{1}$ Survey of Medicinal Plants Unit (S), CCRS, Salem, Tamil Nadu - 636 401, India \\ ${ }^{2}$ Department of Botany, Rani Anna Government College for Women, Tirunelveli, Tamil Nadu - 627 008, India \\ 3Department of Botany, The MDT Hindu College, Tirunelveli, Tamil Nadu - 627 010, India \\ *Corresponding author; e-mail: saran_gan@rediffmail.com
}

\begin{tabular}{|c|c|}
\hline Article Info & ABSTRACT \\
\hline $\begin{array}{l}\text { Date of Acceptance: } \\
20 \text { May } 2020\end{array}$ & \multirow{9}{*}{$\begin{array}{l}\text { Survey at Point Calimere Wildlife and Bird Sanctuary revealed six groves were located } \\
\text { in the area of study. There is diversity in deities worshipped. The selected groves show } \\
\text { the existence of many species of medicinal plants. In those six groves, } 210 \text { species of } \\
\text { flowering plants were found. Salvadora persica is classified as 'Least Concern' in the } \\
\text { IUCN Red List of Threatened Species 2019. Drosera indica and Drosera burmanni are } \\
\text { carnivorous plants used extensively in herbal medicine and are classified as 'Least } \\
\text { Concern' in the IUCN Red List of Threatened Species 2013. Commiphora berryi is a } \\
\text { peninsular endemic species recorded in the present study. This wetland sacred forest } \\
\text { area is of high biodiversity, with many unique species of animals and birds. The loss of } \\
\text { faith, extraction of timber, shifting of deity/restricting the abode of the deity, clearing of } \\
\text { groves for development activities, Biomass removal, Poaching and migration and } \\
\text { occupation of lands by people of other faiths are the existing threats and major reasons } \\
\text { for the decline of sacred groves. }\end{array}$} \\
\hline Dat & \\
\hline & \\
\hline Keywords & \\
\hline Biodiversity & \\
\hline Deity & \\
\hline & \\
\hline In-situ conservation & \\
\hline Sacred groves & \\
\hline
\end{tabular}

\section{Introduction}

Many traditional conservation practices of indigenous people in many parts of the world such as protection of small forest patches by dedicating them to the local deity, also contributed to the conservation and protection of biodiversity. Such forest patches called 'Sacred groves' are tracts of virgin forest harbouring rich biodiversity, protected by the local people based on their indigenous cultural and religious beliefs and taboos. Indigenous knowledge systems or traditional practices of different communities are as valuable as they are the source of solution to many present-day problems. Sacred groves (SGs) in India refer to tracts of usually virgin forests of varying sizes which are communally protected having a significant religious connotation for protecting the community (Khan et al., 2008). Biologically, they are rich patches of undisturbed forests and serve as a natural habitat for many endemic, rare, primitive and economically valuable plants along with a good number of wild animals, birds, reptiles, amphibians, variety of butterflies and insects (Samati and Gogoi, 2007). In India, about 13,720 sacred groves have been enumerated from different states (Malhotra et al. 2001). There are about 235 Sacred Groves recorded from 
Eastern Ghats districts of Tamil Nadu (Swain et al. 2008). The present study was on the sacred groves of the Point Calimere Wildlife and Bird Sanctuary, Tamil Nadu, India.

The following are the specific objectives of the study.

- To conduct a survey of the sacred groves in Point Calimere Wildlife and Bird Sanctuary.

- To list the external influences leading to the degradation of sacred groves.

\section{Materials and methods}

Preliminary survey for collecting data on various aspects about the sacred grove was attempted. Survey of flora was made in selected groves by total inventory method. The source of materials for this floristic research was the extensive field collections of specimens made from the area of study during the period from Apr. 2010 to June. 2011. During the course of this study 14 field trips were undertaken. Standard methodology was used to elicit the knowledge of sacred grove plants. All the relevant information of each sacred grove plants was recorded. The plants specimens collected were processed at the Survey of Medicinal Plants Unit Siddha (CCRS), Mettur Dam, Salem district, Tamil $\mathrm{Nadu}$ and identified with the help of available literature. The collected specimens were identified and authenticated with the help of valid references (Gamble and Fischer, 1921-1935; Nair and Henry, 1983; Matthew, 1983; Daniel and Umamaheswari, 2001). Photographs of the area of study were taken with Pentax - K 1000, Canon - Power shot A710 IS camera in order to depict the contour and other important locality features. Oral history about the sacred groves was collected from elder generation to document the change that has taken place within a specific period.

\section{Results and discussion}

\section{Sacred groves in Point Calimere}

Point Calimere is associated with the Hindu epic, The Ramayana. The highest point of the cape, at an elevation of $4 \mathrm{~m}$, is Ramarpadam, "Rama's feet" in Tamil (Fig. 1a and 1b). A stone slab bears the impressions of two feet and is understood to be the place where Rama stood and reconnoitred Ravana's kingdom in Sri Lanka. Large number of Rama devotees gathers here during the $2^{\text {nd }}$ week of April to celebrate the Ram Navami Festival. Modi Mandapam is a shrine located near Ramarpadam (Fig. 1c). Hindu legend says that Lord Vedaraneswarer spends a night here with his consort during January - February. In the first week of March a major festival is held here. Sanyasi Muniaswar Kovil is a shrine between the eastern bank of Muniappan Lake and Kodikkarai road visited by devotees on all auspicious occasions. On March 20 a special Puja is celebrated here. Mattumunian Kovil (Fig. 1d and $1 \mathrm{e}$ ) is a small temple in the south of the sanctuary where people worship and offer prayers throughout the year. A major festival is celebrated here on the $3^{\text {rd }}$ Friday of September every year. Avulaiganni Dargah is the grave of a Muslim saint located near the road by Ramarpadam. His death anniversary is observed here at the end of November. Shevrayan Kovil is a shrine to the deities Shevrayan and Soni located deep in the forests of the northern part of the sanctuary. A small village near this shrine was now relocated outside the sanctuary after the creation of Kodikkarai Reserve Forest. Large congregations of devotees from Arcothurai (Akkaraipettai) celebrate a special festival here in June/July every year.

Bathing in the sea at Point Calimere is considered sacred by Hindus and a temple there is an object of pilgrimage. People from Tanjore and Nagapatinum districts visit this area on Adi amavasai (off moon day in the month of Tamil adi) and Thai amavasai (off moon day in the month of Tamil Tai) to pay rituals in remembrance of their departed souls. Sacred groves exist as holy places for worshiping Gods and Goddesses. Many of them have small shrines attached to them but Vanathurkai temple (Fig. 1g) has only a small holy place in the form of idol and a single tree for worship. Complex rituals are performed in almost all groves. Karuppana Samy and Karthavarayan are the prominent deities of male gods and Sri Gomathiamman is the famous female deity (Fig. 1f)). The Kodikkadu temple Kuzhagar Kovil (Fig. 1h) is a Vaishnava temple and the deity is Kuzhazhagar. These temples and Dargah show that this area has a great religious significance. 

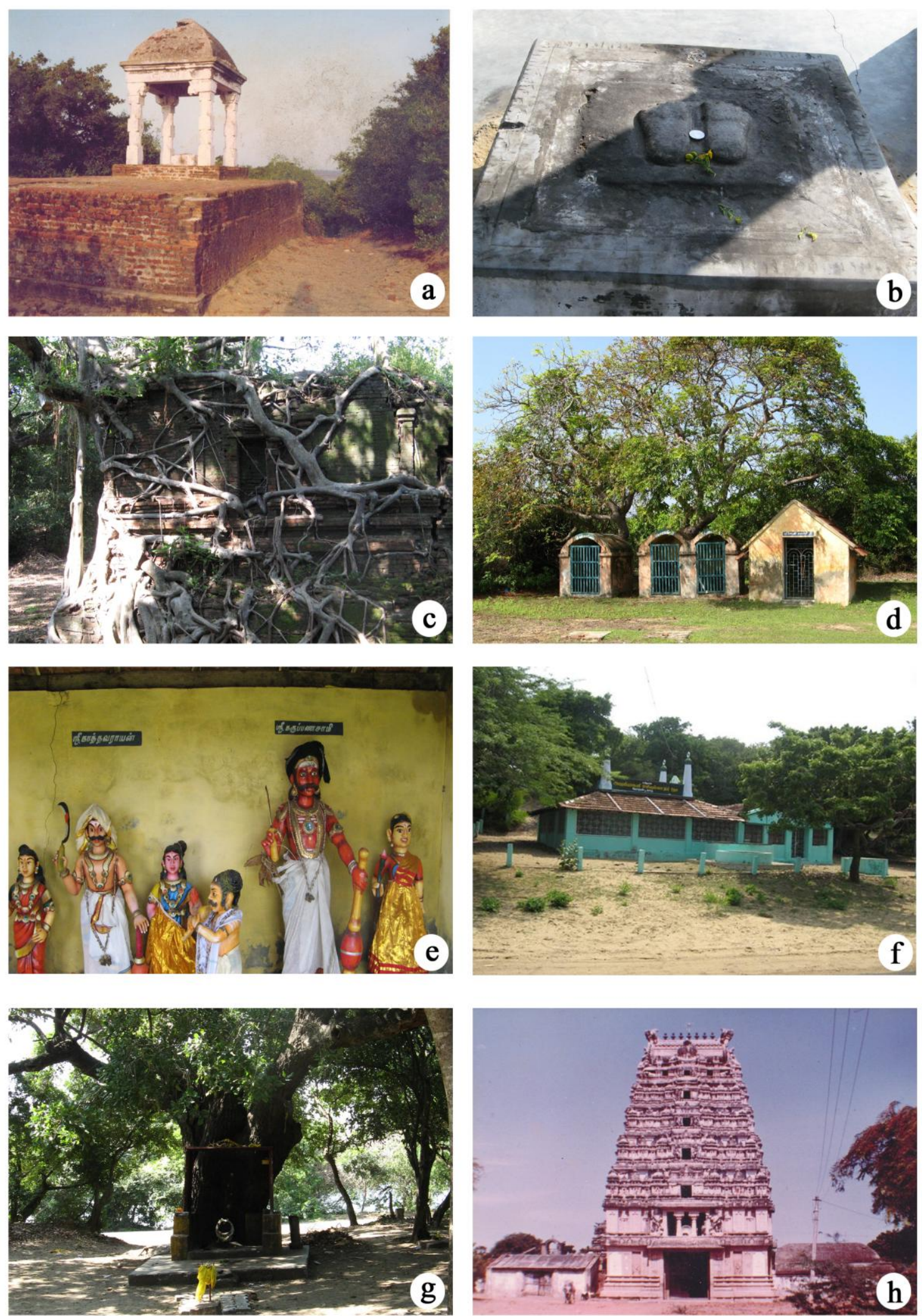

Fig. 1: Sacred Groves at Point Calimere. a: Aerial view of the Ramarpatham at Point Calimere. b: A close up view of the "Rama's feet" c: A view of the badly damaged Modi Mandapam d: Deities of the Mattumunian Kovil; e: Avulaiganni Dargah - the grave of a Muslim saint f: A view of the Avulaiganni Dargah; g: A view of the Vanathurkai temple h: The aerial view of the Kuzhagar koil. 
Table 1. List of plant species recorded in the Point Calimere Wildlife and Bird Sanctuary, Tamil Nadu, India.

\begin{tabular}{|c|c|c|c|c|}
\hline S.no. & Botanical Name & Family & Habit & Vernacular name \\
\hline 1. & Abrus precatorius $\mathrm{L}$. & Fabaceae & Climber & Kundumani \\
\hline 2. & Abutilon indicum (L.) Sweet & Malvaceae & Shrub & Thuthi, Nalla Thuthi \\
\hline 3 . & Acacia nilotica (L.) Willd. ex Delile & Mimosaceae & Tree & Karuvelam \\
\hline 4. & Acalypha fruticosa Forssk. & Euphorbiaceae & Herb & - \\
\hline 5 . & Acalypha indica L. & Euphorbiaceae & Herb & Kuppaimeni \\
\hline 6. & Achyranthes aspera L.W & Amaranthaceae & Herb & Nayuruvi \\
\hline 7. & Aegle marmelos (L.) Correa & Rutaceae & Tree & Vilvam \\
\hline 8. & Aerva lanata (L.) Juss. ex Schult. & Amaranthaceae & Herb & Peelai, Sirupeelai \\
\hline 9. & Ageratum conyzoides L. & Asteraceae & Herb & Poom Pillu \\
\hline 10. & Albizia lebbeck (L.) Willd. & Mimosaceae & Tree & Vaagai \\
\hline 11. & Allmania nodiflora (L.) R. Br. ex Wight & Amaranthaceae & Herb & Vallikeerai \\
\hline 12. & Aloe vera (L.) Burm. f. & Liliaceae & Herb & Chotrukathalai \\
\hline 13. & Alternanthera sessilis (L.) R. Br. ex DC. & Amaranthaceae & Herb & Ponnankanni \\
\hline 14. & Alysicarpus monilifer (L.) DC. & Fabaceae & Herb & Kasukkoti \\
\hline 15 . & Ammannia baccifera $\mathrm{L}$. & Lytharaceae & Herb & Neermel-Neruppu \\
\hline 16. & Anisomeles malabarica (L.)R. Br. ex Sims & Lamiaceae & Herb & Peimiratti \\
\hline 17. & Aristolochia bracteolata Lam. & Aristolochiaceae & Climber & Aduthinnappalai \\
\hline 18. & Aristolochia indica L. & Aristolochiaceae & Climber & $\begin{array}{l}\text { Easwaramooli, } \\
\text { Garudankoti }\end{array}$ \\
\hline 19. & Asparagus racemosus Willd. & Liliaceae & Climber & $\begin{array}{l}\text { Thaneervittan } \\
\text { Kilanzhu }\end{array}$ \\
\hline 20. & Asystasia gangetica (L.) T Anderson & Acanthaceae & Herb & Mitikeerai \\
\hline 21. & Atalantia monophylla (L.) Corr. Serr. & Rutaceae & Tree & Kaattu Elumichai \\
\hline 22. & Azadirachta indica A. Juss. & Meliaceae & Tree & Vaembu \\
\hline 23. & Bacopa monnieri (L.) Pennell & Scorphulariaceae & Herb & Neerbrahmi \\
\hline 24. & Basilicum polystachyon (L.) Moench & Lamiaceae & Herb & Sannaki Poondu \\
\hline 25. & Benkara malabarica (Lam.) Tiruvengadam & Rubiaceae & Shrub & Pudan \\
\hline 26. & Bidens pilosa L. & Asteraceae & Herb & Mukkuthi \\
\hline 27. & Biophytum sensitivum (L.) DC. & Oxalidaceae & Herb & Jala Pushpam \\
\hline 28. & Blumea obliqua (L.) Druce & Asteraceae & Herb & - \\
\hline 29. & Boerhavia diffusa $\mathrm{L}$. & Nyctaginaceae & Herb & Mookarattai \\
\hline 30. & Borassus flabellifer L. & Aracaceae & Tree & Panai \\
\hline 31. & Breynia vitis-idaea (Burm. f.) C.B.C. Fisch & Cucurbitaceae & Shrub & Kattuniruri \\
\hline 32. & Cadaba fruticosa (L.) Druce & Capparaceae & Shrub & Vizhudhi \\
\hline 33 . & Calophyllum inophyllum L. & Clusiaceae & Tree & Punnai \\
\hline 34. & Calotropis giganteana (L.) R. Br. & Asclepiadaceae & Shrub & Erukku \\
\hline 35 . & Canavalia virosa (Roxb.) Wight \&Arn. & Fabaceae & Climber & Kattuttambattan \\
\hline 36. & Canthium parviflorum Lam. & Rubiaceae & Shrub & Kaarai \\
\hline 37. & Capparis sepiaria L. & Capparaceae & Shrub & Karunchurai \\
\hline 38. & Capparis zeylanica L. & Capparaceae & Shrub & Athondai \\
\hline 39. & Cardiospermum halicacabum $\mathrm{L}$. & Sapindaceae & Climber & Mudakotthan \\
\hline 40. & Carissa spinarum L. & Rubiaceae & Shrub & Sirukala \\
\hline 41. & Cassia absus L. & Ceasalpiniaceae & Herb & - \\
\hline 42. & Cassia auriculata $\mathrm{L}$. & Ceasalpiniaceae & Shrub & Avarai \\
\hline 43. & Cassia occidentalis L. & Ceasalpiniaceae & Herb & Peiyavarai \\
\hline 44. & Cassia roxburghii DC. & Ceasalpiniaceae & Tree & - \\
\hline 45 . & Cassia tora $\mathrm{L}$. & Ceasalpiniaceae & Herb & Thagarai \\
\hline 46. & Cathranthus roseus (L.) G. Don & Apocynaceae & Herb & Nithyakalyani \\
\hline 47. & Catunaregam spinosa (Thunb.) Tirveng. & Rubiaceae & Shrub & Madukkarai \\
\hline 48. & Celosia argentea $\mathrm{L}$. & Amaranthaceae & Herb & Pannai keerai \\
\hline 49. & Celosia polygonoides Retz. & Amaranthaceae & Herb & - \\
\hline 50. & Centella asiatica (L.) Urb. & Apiaceae & Herb & Vallarai \\
\hline 51. & Chloris barbata Sw. & Poaceae & Herb & Kodaipullu \\
\hline 52. & Cissampleos pareira L. & Minispermaceae & Climber & Appatta, Puttittiruppi \\
\hline 53. & Cissus quadrangularis $\mathrm{L}$. & Vitaceae & Climber & Pirandai \\
\hline
\end{tabular}




\begin{tabular}{|c|c|c|c|c|}
\hline S.no. & Botanical Name & Family & Habit & Vernacular name \\
\hline 54. & Cleome aspera J. Koenig ex DC. & Capparaceae & Herb & - \\
\hline 55 . & Cleome gynandra $\mathrm{L}$. & Capparaceae & Herb & Nal Vaelai \\
\hline 56. & Cleome viscosa $\mathrm{L}$. & Capparaceae & Herb & Kaattu Kaduku \\
\hline 57. & Clerodendrum inerme (L.) Gaertn. & Verbanaceae & Shrub & Pinarichanganguppu \\
\hline 58. & Clitoria ternatea $\mathrm{L}$. & Fabaceae & Climber & $\begin{array}{l}\text { Sankupushpam, } \\
\text { Kaakanam }\end{array}$ \\
\hline 59. & Coccinia grandis (L.) Voigt. & Cucurbitaceae & Climber & Kovai \\
\hline 60. & Cocculus hirsutus (L.) Diels & Minispermaceae & Climber & Kattukodi \\
\hline 61. & Commelina benghalensis L. & Commelinacea & Herb & Kanaangozhai \\
\hline 62. & Commiphora berryi (Arn.) Engl. & Burusaraceae & Shrub & Mullu Kiluvai \\
\hline 63. & Corchorus trilocularis L. & Tiliaceae & Herb & - \\
\hline 64. & Cordia diffusa K.C. Jacob & Boraginaceae & Herb & - \\
\hline 65. & Crataeva magna (Lour.) DC. & Capparaceae & Tree & Mavilingam \\
\hline 66. & Crotalaria tecta Heyne ex Roth. & Fabaceae & Herb & - \\
\hline 67. & Crotalaria verrucosa $\mathrm{L}$. & Fabaceae & Herb & Cankuniti \\
\hline 68. & Cyanotis cristata (L.) D. Don & Cyperaceae & Herb & - \\
\hline 69. & Cynodon dactylon (L.) Pers. & Poaceae & Herb & Arugam Pullu \\
\hline 70. & Cyperus iria L. & Cyperaceae & Herb & Oosikorai \\
\hline 71. & Cyperus kyllingia Endlicher & Cyperaceae & Herb & Velutta Nirbasi \\
\hline 72. & Cyperus rotundus $\mathrm{L}$. & Cyperaceae & Herb & Koraikilangu \\
\hline 73. & Datura innoxia Mill. & Solanaceae & Shrub & Oomatthai \\
\hline 74. & Delonix elata (L.) Gamble & Ceasalpiniaceae & Tree & Vaadhanarayanan \\
\hline 75 . & Dendrophthoe falcata (L. f.) Etting. & Loranthaceae & Herb & Pulluruvi \\
\hline 76. & Digera muricata (L.) Mart. & Amaranthaceae & Herb & Thoyya Keerai \\
\hline 77. & Dioscorea pentaphylla L. & Dioscoreaceae & Climber & Kaattuvalli \\
\hline 78. & Dodonaea viscosa (L.) Jacq. & Sapindaceae & Shrub & Virali \\
\hline 79. & Dolichos trilobus L. & Fabaceae & Herb & - \\
\hline 80. & Drosera burmannii Vahl & Droseraceae & Herb & Azhukanni \\
\hline 81. & Drosera indica L. & Droseraceae & Herb & - \\
\hline 82. & Drypetes sepiaria (Wight \&Arn.) Pax Hoffm. & Euphorbiaceae & Tree & Kalvirai \\
\hline 83. & Ecbolium ligustrinum (Vahl) Vollesen & Acanthaceae & Herb & Neelambaram \\
\hline 84. & Eclipta prostrata L. & Acanthaceae & Herb & Vellai Karisalakanni \\
\hline 85. & Elytraria acaulis (L. f.) Lindau & Asteraceae & Herb & Pumikatambam \\
\hline 86. & Emilia sonchifolia (L.) DC. & Asteraceae & Herb & Myualchevi \\
\hline 87. & Enicostema axillare (Lam.) Raynal & Gentianaceae & Herb & Vellarugu \\
\hline 88. & Eragrostis japonica (Thunb.) Trin. & Poaceae & Herb & Kanjirapul \\
\hline 89. & Eriocaulon quinquangulare L. & Eriocaulaceae & Herb & - \\
\hline 90. & Euphorbia hirta L. & Euphorbiaceae & Herb & Ammanpacharisi \\
\hline 91. & Evolvulus alsinoides L. & Convolvulaceae & Herb & Vishnukarandi \\
\hline 92. & Ficus benghalensis L. & Moraceae & Tree & Aal \\
\hline 93. & Ficus microcarpa L. & Moraceae & Tree & - \\
\hline 94. & Ficus religiosa $\mathrm{L}$. & Moraceae & Tree & Arasu \\
\hline 95. & Ficus virens Alton. & Moraceae & Tree & Malai Icchi \\
\hline 96. & Gmelina asiatica $\mathrm{L}$. & Acanthaceae & Shrub & Nilakkumizh \\
\hline 97. & Gomphrena globosa L. & Amaranthaceae & Herb & Vatamalli \\
\hline 98. & Grewia umbellifera Beddome & Tiliaceae & Shrub & - \\
\hline 99. & Hedyotis corymbosa (L.) Lam. & Rubiaceae & Herb & Kattukkayaver \\
\hline 100. & Heliotropium indicum L. & Boraginaceae & Herb & Thaelkodukku \\
\hline 101. & Heliotropium marifolium Retz. & Boraginaceae & Herb & - \\
\hline 102. & Hemisdesmus indicus (L.) R.Br. var. indicus & Asclepiadaceae & Climber & Nannaari \\
\hline 103. & Hybanthus enneaspermus (L.) F. Muell. & Violaceae & Herb & Orilai Thamarai \\
\hline 104. & $\begin{array}{l}\text { Hygrophila schulli (Buch-Ham.) M. R. \& S.M. } \\
\text { Almeids }\end{array}$ & Acanthaceae & Herb & Neermulli \\
\hline 105. & Hyptis suaveolens (L.) Poir. & Lamiaceae & Herb & - \\
\hline 106. & Indigofera aspalathoidesVahl ex DC. & Fabaceae & Herb & Sivanaar Vaembu \\
\hline
\end{tabular}




\begin{tabular}{|c|c|c|c|c|}
\hline S.no. & Botanical Name & Family & Habit & Vernacular name \\
\hline 107. & Indigofera linnaei Ali & Fabaceae & Herb & Sheppunerunji \\
\hline 108. & Ipomoea obscura (L.) Ker. & Convolvulaceae & Climber & - \\
\hline 109. & Ixora pavetta Andr. & Rubiaceae & Shrub & Culundu \\
\hline 110. & Jasminum officinale L. & Oleaceae & Climber & Malligai \\
\hline 111. & Jasminum sambac (L.) Ait. & Oleaceae & Climber & Malligai \\
\hline 112. & Justicia adhatoda L. & Acanthaceae & Shrub & Adhatoda \\
\hline 113. & Lawsonia inermis $\mathrm{L}$. & Lytharaceae & Shrub & Maruthondri \\
\hline 114. & Leucas aspera (Willd.) Link & Lamiaceae & Herb & Thumbai \\
\hline 115. & Limonia acidissima $\mathrm{L}$. & Rutaceae & Tree & Villa Maram \\
\hline 116. & Lindernia antipoda (L.) Alston & Scorphulariaceae & Herb & Thanneer Poondu \\
\hline 117. & Ludwigia perennis L. & Onagraceae & Herb & \\
\hline 118. & $\begin{array}{l}\text { Madhuca longifolia (Koen.) Macbr. var. latifolia } \\
\text { (Roxb,) A.Cheval }\end{array}$ & Sapotaceae & Tree & Illupai \\
\hline 119. & Manilkara hexandra (Roxb.) Dubard & Sapotaceae & Tree & Ulakkai-P-Paalai \\
\hline 120. & Melochia corchorifolia L. & Sterculiaceae & Herb & Punnakku Poondu \\
\hline 121. & Memecylon umbellatum Burm. F. & Melastomataceae & Tree & Alli, Anjani \\
\hline 122. & Mimosa pudica L. & Mimosaceae & Herb & Thotaal Surungi \\
\hline 123. & Mimusops elengi L. & Sapotaceae & Tree & Magizham \\
\hline 124. & Mollugo cerviana (L.) Ser. & Molluginaceae & Herb & Parpaadagam \\
\hline 125. & Mollugo nudicaulis Lam. & Molluginaceae & Herb & - \\
\hline 126. & Mollugo pentaphylla L. & Molluginaceae & Herb & Parpaadagam \\
\hline 127. & Momordica dioica Roxb. ex Willd. & Cucurbitaceae & Climber & Pazhupagal \\
\hline 128. & Morinda pubescens $\mathrm{Sm}$. & Rubiaceae & Tree & Nuna \\
\hline 129. & Mucuna pruriens (L.) DC. & Fabaceae & Herb & Poonaikkaali \\
\hline 130. & Mukia maderaspatana (L.) M. Roem. & Cucurbitaceae & Climber & Musumusukkai \\
\hline 131. & Nerium oleander L. & Apocyanceae & Shrub & Aralli \\
\hline 132. & Nothosaerva brachiata (L.) Wight, & Amaranthaceae & Herb & - \\
\hline 133. & Ochna obtusata DC. var. obtusata & Ochnaceae & Shrub & Chilanti \\
\hline 134. & Ocimum americanum L. & Lamiaceae & Herb & Nai Thulasi \\
\hline 135. & Ocimum tenuiflorum $\mathrm{L}$. & Lamiaceae & Herb & Nala Thulasi \\
\hline 136. & Passiflora foetida L. & Passifloraceae & Climber & Siruppoonaikkaali \\
\hline 137. & Pavetta indica $\mathrm{L}$. & Rubiaceae & Tree & Pavattai \\
\hline 138. & Pavonia odorata Willd. & Malvaceae & Herb & Peramutti \\
\hline 139. & Pedalium murex $\mathrm{L}$. & Pedaliaceae & Herb & Perunerunji \\
\hline 140. & Pentatropis capensis (L.f.) Bullock & Asclepiadaceae & Climber & Oopilakodi \\
\hline 141. & Pergularia daemia (Forssk.) Chiov. & Asclepiadaceae & Climber & Vaeliparuthi \\
\hline 142. & Peristrophe paniculata (Forssk.) Brummit & Acanthaceae & Herb & Kara-K-Kanchiram \\
\hline 143. & Phyla nodiflora (L.) Greene & Verbenaceae & Herb & Poduthalai \\
\hline 144. & Phyllanthus amarus Schum. \&Thonn. & Euphorbiaceae & Herb & Keezhanelli \\
\hline 145 . & Phyllanthus emblica L. & Euphorbiaceae & Herb & Nelli \\
\hline 146. & Phyllanthus maderaspatensis $\mathrm{L}$. & Euphorbiaceae & Herb & Melanelli \\
\hline 147. & Phyllanthus rotundifolius Klein ex Willd & Euphorbiaceae & Herb & Thengaipoo \\
\hline 148. & Physalis minima L. & Malvaceae & Herb & Tholthakkali \\
\hline 149. & Pisonia grandis $\mathrm{R}$. Br. & Nyctaginaceae & Tree & Illachikkettailai \\
\hline 150. & Pleurostylia opposita (Wall.) Alston & Celastraceae & Tree & Chiru Piyari, Karuvali \\
\hline 151. & Polycarpaea corymbosa (L.) Lam. & Caryophyllaceae & Herb & $\begin{array}{l}\text { Nilaichedaichi, } \\
\text { Katacciver }\end{array}$ \\
\hline 152. & Polygala arvensis Willd. & Polygalaceae & Herb & Milakunankai \\
\hline 153. & Polygala bulbothrix Dunn & Polygalaceae & Herb & - \\
\hline 154. & Polygala javana DC. & Polygalaceae & Herb & Selagachedi \\
\hline 155. & Pongamia pinnata (L.) Pierre & Fabaceae & Climber & Pungam \\
\hline 156. & Portulaca oleracea L. & Portulacaceae & Herb & Paruppu Keerai \\
\hline 157. & Portulaca quadrifida $\mathrm{L}$. & Portulacaceae & Herb & Pasalai Keerai \\
\hline 158. & Premna serratifolia L. & Verbenaceae & Shrub & Pasumunnai \\
\hline 159. & Pupalia lappacea (L.) Juss. & Amaranthaceae & Herb & Aadai-Otti \\
\hline
\end{tabular}




\begin{tabular}{|c|c|c|c|c|}
\hline S.no. & Botanical Name & Family & Habit & Vernacular name \\
\hline 160. & Rivea hypocrateriformis (Desr.) Choisy & Asclepiadaceae & Climber & Boddikkoora \\
\hline 161. & Rothia indica (L.) Druce & Fabaceae & Tree & Nurreypittan Keerai \\
\hline 162. & Ruellia tuberosa L. & Acanthaceae & Herb & Pattaskai \\
\hline 163. & Salacia chinensis L. & Celastraceae & Tree & Pon Korandi \\
\hline 164. & Salvadora persica L. & Salvadoraceae & Tree & Peru-Vila \\
\hline 165. & Sapindus emarginatus Vahl & Sapindaceae & Tree & Poovandikottai \\
\hline 166. & Scilla hyacinthina (Roth) J. F. Macbr. & Liliaceae & Herb & Kattu Velvenkayam \\
\hline 167. & Scoparia dulcis L. & Scrophpulariaceae & Herb & Sarakkotthini \\
\hline 168. & Securinega leucopyrus (Wild.) Mill. & Euphorbiaceae & Herb & Varat-Pula \\
\hline 169. & Sida acuta Burm. f. & Malvaceae & Herb & Ponmucuttai \\
\hline 170. & Sida cordata (Burm. f.) Borss. Waalk. & Malvaceae & Herb & Kurunthotti \\
\hline 171. & Sida cordifolia $\mathrm{L}$. & Malvaceae & Herb & Chittamutti \\
\hline 172. & Solanum americanum Mill. & Solanaceae & Herb & Manathakali \\
\hline 173. & Solanum tribolatum $\mathrm{L}$. & Solanaceae & Climber & Thuthuvalai \\
\hline 174. & Sopubia delphinifolia (L.) G. Don. & Scrophpulariaceae & Herb & - \\
\hline 175. & Spermacoce hispida L. & Rubiaceae & Herb & Naththaisuuri: \\
\hline 176. & Sphaeranthus indicus L. & Astreraceae & Herb & Kottai Karanthai \\
\hline 177. & Strebulus asper Lour. & Moraceae & Tree & Piray \\
\hline 178. & Striga asiatica (L.) Kuntze & Scrophpulariaceae & Herb & Pallipoondu, \\
\hline 179. & Strychnos minor Dennst & Loganiaceae & Tree & Kodi Etti \\
\hline 180. & Synedrella nodiflora (L.) Gaertn, & Asteraceae & Herb & $\begin{array}{l}\text { Mudiyan } \\
\text { Achchai }\end{array}$ \\
\hline 181. & Syzygium cumini (L.) Skeels & Myrtaceae & Tree & Naval \\
\hline 182. & Tarenna asiatica (L.) Kuntze ex K.Schum & Rubiaceae & Tree & Pavattachedi \\
\hline 183. & Tephrosia maxima Pers. & Fabaceae & Herb & - \\
\hline 184. & Tephrosia purpurea (L.) Pers. & Fabaceae & Herb & Kolunchi. \\
\hline 185. & Terminalia arjuna (Roxb. ex DC.) Wight \& Arn. & Combretaceae & Tree & Marutu \\
\hline 186. & Terminalia catappa $\mathrm{L}$. & Combretaceae & Tree & Nattuvadumai \\
\hline 187. & Thespesia populnea (L.) Sol. ex Correa & Malvaceae & Tree & Puvarasu \\
\hline 188. & $\begin{array}{l}\text { Tinospora cordifolia (Willd.) Miers ex Hook f. } \\
\text { \&Thoms. }\end{array}$ & Minispermaceae & Climber & Seendil Kodi. \\
\hline 189. & Toddalia asiatica (L.) Lam. & Rutaceae & Shrub & Kattu-Milaku \\
\hline 190. & Tranthema portulacastrum L. & Aizoaceae & Herb & Sharunnai \\
\hline 191. & Tribulus terrestris L. & Zygophyllaceae & Herb & Nerunji Mull \\
\hline 192. & Trichodesma indicum (L.) R. Br. & Boraginaceae & Herb & Kiluttaitumpai, \\
\hline 193. & Trichosanthes cucumerina L. & Cucurbitaceae & Climber & Kattuppeypputal \\
\hline 194. & Trichosanthes tricuspidata Lour. & Cucurbitaceae & Climber & Kurattai \\
\hline 195. & Tridax procumbens L. & Asteraceae & Herb & Vettukkaaya-Thalai \\
\hline 196. & Triumfetta rhomboidea Jacq. & Tiliaceae & Herb & Puramutti \\
\hline 197. & Triumfetta rotundifolia Lam. & Tiliaceae & Herb & Adayotti \\
\hline 198. & Tylophora indica (Burm. F) Merr. & Asclepiadaceae & Climber & Nancharuppan \\
\hline 199. & Urginea indica (Roxb.) Kunth & Liliaceae & Herb & Nari-Venkayam \\
\hline 200. & Vernonia cinerea (L.) Less & Asteraceae & Herb & Naichotte Poondu \\
\hline 201. & Vicoa indica (L.) DC. & Asteraceae & Herb & - \\
\hline 202. & Vitex negundo L. & Verbanaceae & Shrub & Nocchi \\
\hline 203. & Walsura trifolia $\mathrm{L}$. & Meliaceae & Tree & Kanjimaram \\
\hline 204. & Waltheria indica $\mathrm{L}$. & Sterculiaceae & Herb & - \\
\hline 205. & Wattakaka volubilis (L. f.) Stapf. & Asclepiadaceae & Climber & Ankara-Valli \\
\hline 206. & Wrightia tinctoria (Roxb.) R. Br. & Rubiaceae & Tree & Veppalai \\
\hline 207. & Xanthium indicum J. Konig & Asteraceae & Herb & Maruluummatti \\
\hline 208. & Ziziphus oenoplia (L.) Mill. & Rhmnaceae & Tree & Surai Ilantai \\
\hline 209. & Ziziphus xylopyrus (Retz.) Willd. & Rhmnaceae & Tree & Kottai-Elandai \\
\hline 210. & Zornia diphylla (L.) Pers. & Fabaceae & Herb & Chirupalatai \\
\hline
\end{tabular}


There is diversity in deities worshipped. Each has a presiding deity and most of them have associated deities too. Presiding deity may be a God, Goddess or Serpent. Snake is also the associated deity in some sacred groves.

The rituals represent a mixture of the practices of an agrarian society and a hunting society. There are folklores explaining the power of each deity. Folklores have evolved into myths which compel the society to worship deities and to perform rituals. Strong taboos prevented the society from exploiting the resources of the sacred groves.

\section{Vegetation of the Sacred Groves}

The sacred forests of this region are ecologically better preserved. The forest type is mostly of wet evergreen. A few have drier types ranging from semi-evergreen to deciduous. All maintain fertility of soil and purity and humidity of air in the surroundings. The neighbouring lands get water from them. They are the centres of village biodiversity.

Our survey revealed the abundance of sacred groves in this area. Six groves are located in the area of study. The groves are having size of 5 cents. The tropical climate of the locality supports growth of evergreen species, resulting in the majority of the groves having evergreen vegetation. The Kodikkadu temple grove is semi-evergreen. The area consists of stunted, almost exclusively formed of bushed much branched plant species measuring 2 to $4 \mathrm{~m}$ in height. Here and there emerge some evergreen arborescent shrubs under $10 \mathrm{~m}$ in height with dark green voluminous crowns. Being accessible, this vegetation is often traversed by man and openings are made in places. Also, some anthropic stages appear here and there with anthropochore trees like Syzygium cumini, Borassus flabellifer, Lannea coromandelica and Manilkara hexandra.

This is the most productive vegetation of the forest in terms of biomass as well as utility. Dendroid forms of Manilkara, Calophyllum, Walsura, Syzygium, Dryptes and climbers or stragglers like Mucuna, Canavalia, Salacia, Cissus, Toddalia, Tinospora occur here are valuable as food and medicine.
The six groves support 210 species of angiosperms covering 172 genera under 64 families. Herbs, shrubs, trees and climbers represent 117, 25, 39 and 29 species respectively. It bears species with edible fruits, species having sacred value, species used as timber plants, species having firewood and medicinal values, of which a few are rapidly vanishing from the surrounding forest areas. In dicots 195 species are representing 58 families. Among the dicots, family Fabaceae is the most dominant with 15 species. Family Rubiaceae and Asteraceae are represented by 11 species each. In Monocot 15 species are representing 6 families. Among the monocots, the most dominant family is Cyperaceae (4 species). One species of Lichen (Rocella), two species of Pteridophytes (Marselia) and one species of Bryophyte (Riccia) are also recorded in the area of study. Insectivores plants like Drosera burmannii, and Drosera indica have been recorded in the area of study. Dryptes sepiaria and Manilkara hexandra, Ficus microcarpa and Aegle marmelos are treated most important as sacred trees. Lord Vanathurka is placed under the Manilkara hexandra tree, which is around 200 years old having around $2 \mathrm{mt}$. circumferences. Dendrophthoe falcata is a parasite on tree species like Azhadirachta, Sapindus etc. During the rainy season, aquatic ephemerals such as Ludwigia Lindernia, Bacopa, Marsilea etc., come up. Prosopis an introduced plant is progressively encircling patches of natural vegetation and strangles them extending inwards.

Salvadora persica is classified as 'Least Concern' in the IUCN Red List of Threatened Species 2019. Drosera indica and Drosera burmanni are carnivorus plants used extensively in herbal medicine and are also classified as 'Least Concern' in the IUCN Red List of Threatened Species 2013. The study reported Breynia vitis-idaea is exclusively insular, based on the literature available in Flora of Gulf of Mannar (Daniel and Umamaheshwari, 2001). Commiphora berryi is a peninsular endemic species recorded in the present study. Cleome aspera, Corallacarpus epigaeus, Indigofera aspalathoides, Pentatropis capensis, Sopubia delphinifolia, Terminalia arjuna, Zizyphus mauritiana and $Z$. Xylopyrus are distributed exclusively to Sri Lanka and Peninsular India as cited in the Flora of Gulf of Mannar (Daniel and Umamaheshwari, 2001). 


\section{Ecological significance of sacred groves}

From time immemorial, the sacred groves in India have been the focus and symbol of a way of life. In such groves the highest levels of biological diversity are still found; there humans interact with nature. Ecologically valuable species function as keystone species in an ecosystem and contribute to the enhancement of biodiversity (Ramakrishnan, 1996). They are also species that are socially valued by the local village communities for cultural and religious reasons. The present investigation reveals that each grove is maintaining a microclimate with rich biodiversity. The selected groves show the existence of many species of medicinal plants. In the six groves, 210 species of flowering plants were recorded. The abundant and widely distributed families are Fabaecae, Rubiaceae, Euphorbiaceae and Moraceae. Very common genera are Sida acuta, Abrus precatorius, Acanthus ilicifolius, Phyllanthus amarus, Drosera burmannii, Drosera indica and Ocimum tenuiflorum. Large trees such as Dryptes sepiaria, Manilkara hexandra, Ficus racemosa, Sapindus emarginatus, Salvadora persica, Syzygium cuminii, Aegle marmelos and Walsura trifolia are the other species found in the groves. Another remarkable feature of the groves is the luxuriant growth of Cressa cretica and Eriocaulon quinquangulare. Medicinal plants like Aegle marmelos, Phylllanthus emblica, Tinospora cordifolia, Capparis zeylanica, Mucuna pruriens, Asparagus racemosus, Solanum trilobatum, Phyllanthus amarus and Aloe vera are very common in groves. From the floristic study it is clear that these sacred groves are the remarkable treasure-houses of biodiversity of Point Calimere. So, from the conservation point of view, these groves are most useful for in situ conservation of biodiversity.

The general phytosociology of sacred grove is extremely complex. Woody plants are the dominant species which include trees and lianas. The sacred groves of Point Calimere show remarkable physiognomic features in stratification, girth class, presence of epiphytes, vines, lianas, etc., due to the specific characteristics of the locality and the various degrees of interference made by the associated fauna and by man. The three strata of trees, shrubs and herbs, climbers and stragglers, epiphytes and parasites are the floristic elements of a grove. Here, the upper canopy includes trees with straight and slender trunk and branches are produced at the top. Common emergent tree species are Manilkara hexandra, Syzygium cumini, Calophyllum inophyllum, Pleurostylia opposita, Sapindus emarginatus, Ficus racemosa and Walsura trifolia. The second stratum includes members like Mimosops elengi, Canthium dicoccum, Ochna obtusata and Cassia fistula. The lower stratum consists of species like Memecylon umbellatum, Todallia asiatica, Gmelina asiatica, Capparis sepiaria and Dryptes sepiaria. Due to the massive growth of spreading climbers like Abrus precatorius, Asystasia gangetica, Tinospora cordifolia, Trichosanthes cucumerina, Mucuna pruriens, Cappariis zeylanica, Canavalia virosa and Salacia chinensis the canopy is more or less continuous. The herbaceous flora in a sacred grove is rain-dependent and most of them are annuals. The number of herbaceous plants is less in dry season. The density and species composition appearance of herbaceous flora is according to their demand for light. Luxuriant herbaceous vegetation is found where the canopy is open.

Sacred groves preserve biodiversity within their boundaries. Now they are under threat. The loss of faith, extraction of timber, shifting of deity/restricting the abode of the deity, clearing of groves for development activities, biomass removal, poaching and migration and occupation of lands by people of other faiths are the existing threats and major reasons for the decline of sacred groves. Some of the families settled nearby were dependent on the forests for their energy requirement. A part of this was collected from this grove. Despite the high reverence for this forest and regulations attached, the firewood collectors, who were mostly identified as non-locals, did not spare the grove. This was another added challenge for the long-term survival of this natural repository. Continuous fodder collection was noticed as a prominent threat. Most rural populace owned some grazing animal for which large quantities of forage were harvested from this grove. A number of wild edibles were abundance in the grove. Locals were making collections even during this survey. This process could adversely impact the rates of natural regeneration of many species of plants that serve the double purpose of being useful to man and some faunal species. 
Conservation of biodiversity and maintenance of the eco-system is of overriding importance for the survival of the human race itself. The sacred groves have played a role in that endeavour. Those benefits might, however, soon be lost if they are allowed to be degraded or destroyed. Thus, protection of sacred groves must gain priority over other less important goals. Further detailed exploration of sacred groves in Tamil Nadu is an immediate requirement to assess their composition, various threat factors and conservation potential for their existence. Such gene pool reserves can definitely serve as icons of in situ conservation under the prevailing times through a good mix of scientific measures and awareness building efforts with the active involvement of the local community and the government.

\section{Conflict of interest statement}

Authors declare that they have no conflict of interest.

\section{Acknowledgement}

The authors are thankful to Dr. K. Natarajan, Head of the Department of Botany (Rtd.), St. Xavier's College, Palayamkottai, Tirunelveli for the constant encouragement and providing facilities.

\section{References}

Daniel, P., Umamaheswari, P., 2001. The Flora of the Gulf of Mannar, Southern India. Botanical Survey of India, Coimbatore.

Gamble, J.S., Fischer C. E. C., 1921-1935. Flora of Presidency of Madras, Vol. I, II and III. London, Adlard and Son Ltd.

Khan, M. L., Ashalata Devi, K., Tripathi R. S., 2008. The sacred groves and their significance in conserving biodiversity - An overview. Int. J. Ecol. Environ. Sci. 34(3), 277-291.

Malhotra, K. C., Gokhle, Y., Chatterjee, S., Srivastava, S., 2001. Cultural and Ecological Dimensions of Sacred Groves in India, INSA, New Delhi.

Matthew, K. M., 1983. Flora of Tamil Nadu Carnatic. The Raphinat Herbarium Tiruchirapalli, India.

Nair, N. C., Henry, A. N., 1983. Flora of Tamil Nadu, India Series 1: Analysis. Vol. I - III. Botanical Survey of India, Coimbatore.

Ramakrishnan, P. S., 1996. Shifting Agriculture and Sustainable Development. MAB Series, UNESCO, Paris.

Samati, H., Gogoi, R., 2007. Sacred Groves in Meghalaya. Curr. Sci. 93(10), 25.

Swain, P. K., Siva Ramakrishnan, I., Murty, B. L. N., 2008. Sacred groves- Their distribution in Eastern Ghats region. EPTRI - ENVIS Newslett. 14(4), 4 .

\section{How to cite this article:}

Padma Sorna Subramanian, M., Saravana Ganthi, A., Subramonian, K., 2020. Sacred groves of Point Calimere Wildlife and Bird Sanctuary - Ideal centres for biodiversity conservation. Int. J. Curr. Res. Biosci. Plant Biol. 7(6), 45-54. doi: https://doi.org/10.20546/ijcrbp.2020.706.005 